International Journal of Computer Networks \& Communications (IJCNC) Vol.3, No.5, Sep 2011

\title{
HYBRID RFID - OWC WITH AN ADAPTIVE Priority TRANSMISSION SCHEME
}

\author{
Wasinee Noonpakdee, Jiang Liu, and Shigeru Shimamoto \\ Graduate School of Global Information and Telecommunication Studies, \\ Waseda University, Tokyo, Japan \\ wasinee@toki.waseda.jp, liujiang@aoni.waseda.jp shima@waseda.jp
}

\begin{abstract}
This paper presents hybrid RFID - OWC with an adaptive priority transmission scheme. In this model, we apply optical wireless communication $(O W C)$ for transmitting confidential information to a radio frequency identification (RFID) reader. Since OWC provides high security, this improves the security of our system. The priority of information is assigned according to the level of confidentiality and significance of the information. The more confidential information is transmitted by OWC with adaptive data rate, and the rest of the information (if there is any) is transmitted by RFID. The energy harvesting of RFID is used to modulate the reflected optical signal by Corner Cube Retroreflector (CCR). Bit error rate $(B E R)$ is analyzed regarding the half-power angle of the transmitter, optical transmit power, data rate, and the angle between tag and reader. The analytical results indicate that by employing the adaptive priority transmission scheme, the required BER of OWC transmission is achieved.
\end{abstract}

\section{KEYWORDS}

RFID, Optical Wireless Communications, image sensors, CCR

\section{INTRODUCTION}

Radio frequency identification (RFID) is a technological tool used for the identification of objects and assets. RFID has attracted extensive interest in recent years because RFID technology has many benefits; for example, non line of sight, contactless, simultaneous collection of data, and high accuracy. RFID systems have been applied in several areas including personal identification, supply chain, food industries, library book tracking, and healthcare. Although RFID systems provide various advantages for several kinds of applications, they have implications for security and privacy concerns [1]-[3]. RFID tags are subject to clandestine (up to few feet) interception, allowing leakage of sensitive personal information [4].

In this paper, we proposed hybrid RFID - OWC with an adaptive priority transmission scheme to improve the security of an RFID system. In our model, the confidential information is transmitted using optical wireless communication (OWC) since OWC provides high security against casual eavesdropping. Optical signals cannot penetrate through walls or other opaque barriers, so the coverage of infrared systems is limited to the boundaries of the room in which the system is installed [5]-[7].

Although OWC has some drawbacks, which includes the possibility of an obstruction in the communication path, restrictions to the emitted optical power for the sake of eye safety and the fact that noise is introduced into the detector from sources of background illumination [8], OWC offers several potential advantages which includes large unregulated bandwidth, DOI : 10.5121/ijcnc.2011.3510 
International Journal of Computer Networks \& Communications (IJCNC) Vol.3, No.5, Sep 2011

immunity to interference caused by other RF wireless devices, possibility of frequency reuse and the use of inexpensive optoelectronic devices which are small and consume little power [9]. Moreover, OWC links are suitable to portable devices since large quantity of small surface mount light emitting and light detecting components are available at relatively low cost [10]. Therefore, OWC is suitable to be applied to our scheme.

In our proposed model, in case the scenarios such as an obstruction existing in the communication path or the bit error rate (BER) of OWC being lower than the required value happen, resulting in the failure of the information transmission from $\mathrm{OWC}$, we reduce the data rate and switch some parts of information that have a lower priority of confidentiality and significance to be transmitted by RFID. Our scheme is applied for a short distance transmission; high SNR is obtained with low transmitted power although OWC system is influenced by noise introduced from background illumination. Moreover, our scheme requires low transmitted power which is much lower than the limit for the average transmitted optical power according to eye safety standard. Therefore, by combining the advantages of RFID and OWC, our proposed model is very promising with high security.

In RFID communication, we focus on the 13.56-MHz inductive passive HF RFID system since HF RFID achieves good performance in a crowded environment [11]. By employing energy harvesting, the RFID tag obtains energy during reader interrogation periods, and the energy is used for Corner Cube Retroreflector (CCR) to demodulate the signal and reflect this signal back to the reader. Our proposed model can be applied in systems that require high security for sending confidential information such as e-passports or identification cards (ID card).

The rest of this paper is organized as follows. In Section 2, the system model is provided. Section 3 presents power estimation and bit error rate. In Section 4, an adaptive priority transmission scheme is proposed. The implementation and analytical results are described in Section 5. Finally, Section 6 concludes the paper.

\section{SYSTEM MODEL}

The system is composed of two parts: tag (transponder) and reader (interrogator), as illustrated in Figure 1. The RFID tag is composed of a microchip connected to an antenna. This tag can be attached to an object, which needs to be uniquely identified. An RFID reader can access the information embedded in the tag. The RFID reader communicates with the RFID tag using radio waves. In our model, an RFID tag is a passive tag that does not have any power source; the power is derived from the radio frequency generated by the reader.

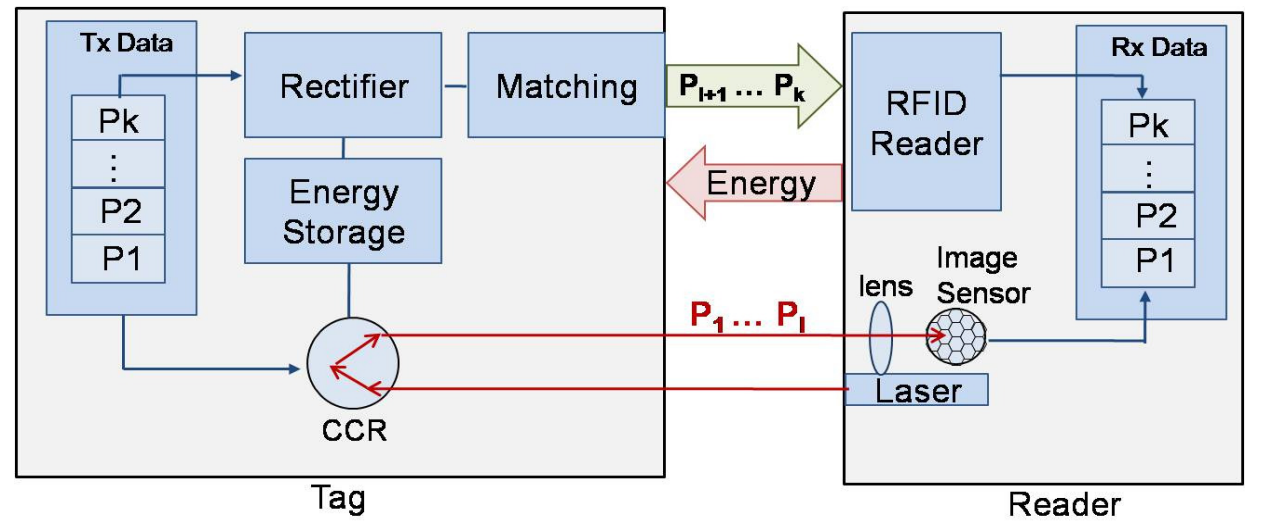

Figure 1. System model 
International Journal of Computer Networks \& Communications (IJCNC) Vol.3, No.5, Sep 2011

We set the OWC transmission devices in both tag and reader. As shown in Figure 1, CCR is placed in the tag to modulate confidential information propagated back to the reader. The reader has a laser source, a lens, and an image sensor. The laser emits optical power, which is limited to protect users' eyes. The optical signal entering the CCR is reflected back to the reader with on-off-keying (OOK), modulated optical signals. At the reader, the optical signal passes through the lens and is received by the image sensor. The imaging design has two advantages over a non-imaging angle-diversity receiver. First, all photo detectors share a common concentrator, thus reducing size and cost. Second, all the photo detectors can be laid out in a single planar array, facilitating the use of a large number of receiving elements or pixels [12].

By applying hybrid RFID-OWC, our model provides high security and the line of sight issues of OWC can be solved. The cost of the proposed system is not so different from the cost of RFID implementation because OWC part can be employed with low cost. Moreover, since the electromagnetic spectrum is not licensed in the optical band, licensing fees are avoided when applying OWC in our system spectrum [10]. Table 1 illustrates the comparison of OWC, RFID, and our proposed hybrid RFID-OWC scheme.

Table 1. Comparison of OWC, RFID, and Hybrid RFID-OWC

\begin{tabular}{|l|c|c|c|}
\hline \multicolumn{1}{|c|}{ Property } & OWC & RFID & Hybrid RFID-OWC \\
\hline Cost & $\$$ & $\$ \$$ & $\$ \$$ \\
\hline Bandwidth Regulation & No & Yes & $\begin{array}{c}\text { No } \\
\text { (when applying OWC) }\end{array}$ \\
\hline Security & High & Low & High \\
\hline Line of sight & Yes & No & No \\
\hline
\end{tabular}

We have adapted our previous work in [13] by employing an adaptive priority transmission scheme. In this scheme, the information is mainly transmitted using OWC. If the BER is higher than the specific value, we reduce the data rate to achieve the specific BER. Then, the portions of the information that are not confidential are transmitted by RFID. At the same time, energy harvesting is processed to apply for CCR to modulate the signal. A CCR offers the advantages of small size, excellent optical performance, low power consumption, and convenient integration with solar cells, sensors, and CMOS control circuits [14].

\section{Power Estimation AND Bit ERror Rate}

In our model, the power can be estimated for the OWC transmission system and the RFID system. In an OWC transmission system, we apply CCR to modulate the confidential data, and transmit this data back by OWC. Signal-to-noise ratio (SNR) and bit error rate (BER) calculation for OWC is described. For an RFID system, in reader interrogation periods, RFID energy harvesting is applied to drive CCR.

\subsection{CCR and optical wireless transmission}

We apply a generalized Lambertian source as an optical transmitter. The reader and tag are in an environment without reflections, and the tag is illuminated with peak intensity. The intensity at CCR $\left(I_{C C R}\right)$ can be estimated as follows:

$$
I_{C C R}=\frac{(n+1)}{2 \pi d^{2}} P_{t} \cos ^{n}\left(\theta_{c}\right) \times \cos \left(\theta_{r}\right) \operatorname{rect}\left(\frac{\theta_{r}}{F O V}\right)
$$

where $d$ is the distance between source and receiver, which is equal to $r / \cos \theta_{c}$. then $r$ is the distance between tag and reader. We assume that the distance between CCR and the laser is the 
International Journal of Computer Networks \& Communications (IJCNC) Vol.3, No.5, Sep 2011

same as the distance between tag and reader. $P_{t}$ is the transmitted power, which is limited to ensure eye safety, $\theta_{c}$ is the angle between the laser and the axis of the link, and $\theta_{r}$ is the angle between the CCR and the axis of the link as illustrated in Figure 2. $\theta_{c}=0^{\circ}$ when the reader is onaxis, and the interrogating beam comes to tag at normal incidence.

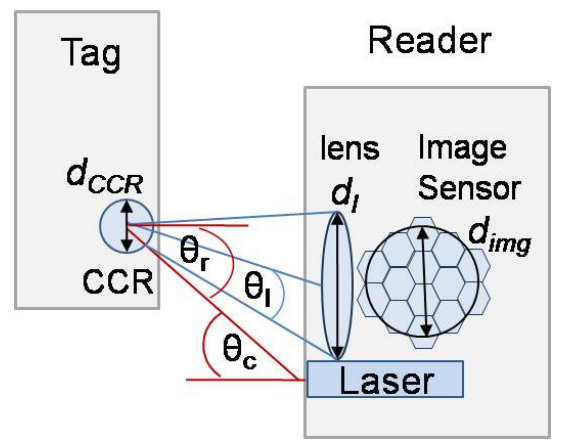

Figure 2. Position of Tag and Reader

FOV is field of view angle, which is the extent of solid-angles from which light is detected by the receiver [13], and $\operatorname{rect}(x)$ is given by

$$
\operatorname{rect}(x)= \begin{cases}1, & |x| \leq 1 \\ 0, & |x|>1\end{cases}
$$

$n$ is the mode number of the radiation lobe that controls the directivity of the specular component of the reflection,

$$
n=\frac{\ln (1 / 2)}{\ln (\cos h p a)}
$$

where $h p a$ is the half-power angle of the transmitter. From (1)-(3), $I_{C C R}$ can be increased by narrowing the transmitter semi-angle, which increases $n$.

Safety considerations must be taken into account when designing an OWC link. Since the energy is propagated in a free-space channel, the impact of this radiation on the human eye and body must be considered. In our system, we apply a safety standard class 1 operation, which is most desirable for a wireless optical system since emissions from products are safe under all circumstances. Under these conditions, no warning labels need to be applied, and the device can be used without special safety precautions. Due to eye safety standards, at wavelength $(\lambda)=$ $880 \mathrm{~nm}$, the average optical power output is less than $0.5 \mathrm{~mW}[10]$.

At tag, we assume that the CCR has effective reflectivity, $R_{C C R}$. The CCR modulates the reflecting optical signal into an OOK signal with non-return-to-zero (NRZ) pulses. Assuming that bit " 0 " and " 1 " are equiprobable, the average power that propagates back from CCR is given by

$$
P_{B}=\frac{I_{C C R} \pi r_{C C R}^{2} R_{C C R}}{2}
$$


International Journal of Computer Networks \& Communications (IJCNC) Vol.3, No.5, Sep 2011

where $r_{C C R}$ is the effective radius of CCR (not tilted). Using the Fraunhofer diffraction theory as in [15], the diffracted irradiance of $i^{\text {th }}$ pixel at the lens that is reflected by CCR is expressed as

$$
I_{l, i}=\frac{P_{B} \pi d_{C C R}^{2} \cos ^{2} \theta_{c} \cos \theta_{l, i}}{4 \lambda^{2} r^{2}}
$$

where $d_{C C R}$ is the effective diameter of CCR (not tilted). $\theta_{l, i}$ represents the angle between the center of the beam and the axis of the link. $\lambda$ represents the interrogation wavelength.

The received photocurrent of $i^{\text {th }}$ pixel at image sensor is calculated by [15]:

$$
I_{i m g, i}=\frac{I_{l, i} \pi d_{l}^{2} T_{l} T_{f} f_{a c t} R}{4}
$$

where $d_{l}$ represents the effective diameter of the lens (not tilted), $T_{l}$ represents the effective transmission of the lens, $T_{f}$ represents the optical filter transmission, $f_{\text {act }}$ represents the fraction of the image sensor pixel area that is active, and $R$ represents the pixel responsivity.

We assume that noise affecting our system is generated at the receiver, and the topology of our system is point-to-point. Consequently, there is no multipath distortion [10], and the intersymbol interference (ISI) is neglected. We consider both shot noise and thermal noise, which are modeled as AWGN with a double-sided power spectral density $=\sigma^{2}$. In this paper, we assume the use of a p-i-n/FET transimpedance receiver [16]. We neglect the noise contributions from gate leakage current and 1/f noise. The expression of the noise variance is given by [16][17]:

$$
\sigma_{t o t, i}^{2}=2 q I_{b g} I_{2} B+\frac{8 \pi k T}{G} \eta A^{\prime} I_{2} B^{2}+\frac{16 \pi^{2} k T_{k} \Gamma}{g_{m}} \eta^{2} A^{\prime 2} I_{3} B^{3}
$$

where $q$ is the electronic charge, $B$ is the equivalent noise bandwidth, $I_{b g}$ is background current, and $I_{2}=0.562$. For the $10 \mathrm{~b} / \mathrm{s}, 100 \mathrm{~b} / \mathrm{s}$, and $1 \mathrm{~kb} / \mathrm{s}$ systems, the bandwidth is limited to $10 \mathrm{~Hz}, 100$ $\mathrm{Hz}$, and $1 \mathrm{kHz}$, respectively [18]. It is possible to use the low data rate to transmit the confidential information since this information is not so large, and the less confidential information can be transmitted by RFID. $k$ is Boltzmann's constant, $T$ is absolute temperature, $G$ is the open-loop voltage gain, $\eta$ is the fixed capacitance of photo detector per unit area, $\Gamma$ is the FET channel noise factor, $g_{m}$ is the FET transconductance, and $I_{3}=0.0868$. We assume the use of an image sensor as in [12], and $A^{\prime}$ is the area of an individual pixel of the image sensor, which is given by this calculation:

$$
A^{\prime}=\frac{3 \sqrt{3}}{8} \frac{d_{\text {img }}^{2}}{\left(\left[\frac{1+\sqrt{1+4 \frac{J-1}{3}}}{2}\right]+\left[\frac{-1+\sqrt{1+4 \frac{J-1}{3}}}{4}\right]\right)^{2}}
$$

where $d_{i m g}$ represents the diameter of the image sensor that is illustrated in Figure 2, and $J$ is the number of equal-sized hexagonal pixels of the receiver area $A_{r} . J$ is calculated by 
International Journal of Computer Networks \& Communications (IJCNC) Vol.3, No.5, Sep 2011

$$
J=2 \sum_{i=1}^{m}(m-i-1)-2 m+1=3\left(m^{2}-m\right)+1
$$

where $m$ is any integer greater than or equal to 2 .

We apply select-best (SB), which represents a simple form of combining techniques by choosing the pixel in the detector array that has the highest signal to noise ratio (SNR). The SNR is calculated by [12]:

$$
S N R=\max \left(\frac{I_{i m g, i}^{2}}{\sigma_{t o t, i}^{2}}\right)
$$

Bit error rate (BER) is obtained by

$$
B E R=Q(\sqrt{S N R})
$$

where $Q(x)=1 / 2 \operatorname{erfc}(x / \sqrt{2})$

\subsection{Harvesting Power of an RFID system}

We apply HF (13.56-MHz) RFID because the operational range of our model is around $0.1-0.5$ $\mathrm{m}$. Since the magnetic field is not affected by most of the surrounding dielectric materials, $\mathrm{HF}$ RFID has good performance in a crowded environment when compared with a UHF (860-960 $\mathrm{MHz}$ ) RFID. In reader interrogation periods, we apply RFID energy harvesting to obtain the energy for driving CCR to modulate the signal. The harvested power $\left(P_{H}\right)$ on the RFID tag IC circuitry can be derived as [11]:

$$
P_{H} \approx \frac{1}{8 R_{t}} \sqrt{\frac{R_{s r}}{R_{r}}} \frac{V_{p}}{2 R_{s r}}\left[\omega M\left(1+\frac{1}{4 R_{r} R_{t}}(\omega M)^{2}\right)^{-1}\right]^{2}
$$

where $R_{t}$ is tag resistance, $R_{r}$ is reader resistance, $R_{s r}$ is source resistance, $V_{p}$ is driven source voltage, $\omega$ is angular frequency, and $M$ is the mutual coupling factor.

From (12), mutual coupling between the reader and the tag affects the amount of power harvested at the tag, and mutual coupling can be viewed as a variable load at the reader. The analytical derivations are confirmed by experimental studies [11].

\section{Adaptive Priority Transmission Scheme}

In our model, we transmit the information mainly by OWC. However, when there is an obstruction in the OWC communication path, or when the reader and tag are not on-axis, the BER will be increased. The flowchart of our proposed scheme is described in Figure 3; if BER is higher than the specific BER that is required for the system $\left(B E R_{r e q}\right)$, we reduce the data rate $\left(R_{b}\right)$ of OWC and transmit some part of the information using RFID. And vice versa, when the BER is lower than the threshold $\left(B E R_{t h}\right)$, the data rate can be increased, and more information can be transmitted by OWC.

As illustrated in Figure 1, the data for transmitting is divided into $P_{1}$ to $P_{k}$ packages depending on the level of confidentiality and significance of the data; for example, the data that is 
International Journal of Computer Networks \& Communications (IJCNC) Vol.3, No.5, Sep 2011

contained in package $\mathrm{P}_{1}$ is more important than the data that is contained in package $\mathrm{P}_{2}$. More confidential or more important data has a higher priority to be transmitted using OWC, which provides higher security than RFID. The data rate is adapted according to the situation of the OWC channel. We limited the data rate to the value between $R_{b_{-} \min }$ and $R_{b_{-} \max }$. Since the data length that is transmitted using our system is not so long, we do not have to implement a high data rate.

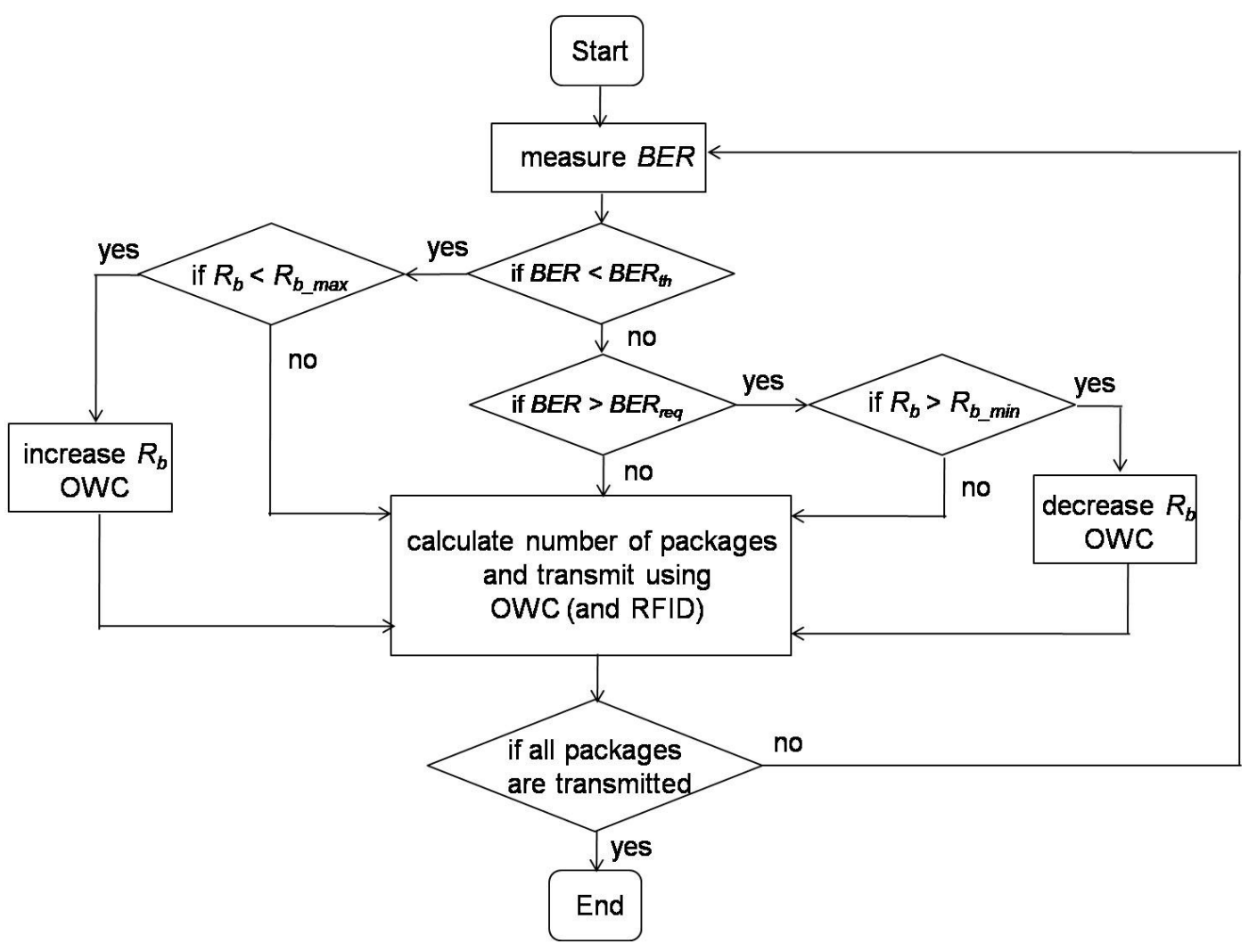

Figure 3. A flowchart of the adaptive priority transmission scheme

\section{IMPLEMENTATION AND ANALYTICAL RESULTS}

In our implementation, RFID power is harvested using (12). We apply the reader antenna with that area of the loop $=0.2 \times 0.2 \mathrm{~m}^{2}$ and one turn, and the tag antenna with the area of the loop = $0.023 \mathrm{~m}^{2}$ and ten turns, $R_{t}=6.2 \Omega, R_{r}=0.5 \Omega, R_{s r}=50 \Omega$, and $V_{p}=5 \mathrm{~V}$. As a result in [11], the harvested power is more than $0.002 \mathrm{~W}$ when the distance of reader and tag is from $0.1-0.5 \mathrm{~m}$. The power obtained from RFID harvesting is used for driving CCR to modulate the signal. Regarding CCR energy consumption, when applying non-return-to-zero (NRZ) encoding, energy is required only when the transmitted bit transitions from " 1 " to " 0 ", so that the average energy consumption is about $19 \mathrm{pJ} / \mathrm{bit}$ [14]. Therefore, the RFID harvesting energy is sufficient for the process of signal modulation at CCR.

When the confidential data is transmitted via OWC, we calculate and analyze SNR and BER with different transmitted power $\left(P_{t}\right)$, data rate $\left(R_{b}\right)$, and angle between tag and reader $\left(\theta_{c}\right)$. Then, we analyze and compare the OWC transmission and the adaptive priority transmission (APT) scheme. We choose the following parameter values [12], [15], [16], as illustrated in Table 2 in our analytical results. 
International Journal of Computer Networks \& Communications (IJCNC) Vol.3, No.5, Sep 2011

In our analytical results, we assume that in order to accomplish a reliable data communication, the BER must be lower than $10^{-4}$, which is $B E R_{\text {req }}=10^{-4}$. We apply $B E R_{t h}=10^{-27}, R_{b_{-} \text {min }}=1 \mathrm{~b} / \mathrm{s}$, and $R_{b_{-} \max }=1 \mathrm{~kb} / \mathrm{s}$. The increase or decrease of the data rate is in the order of $10^{p w}$ where $p w$ is equal to $0,1,2$, or 3 .

Table 2. Analytical Parameters

\begin{tabular}{|c|l|l|}
\hline Symbol & \multicolumn{1}{|c|}{ Description } & \multicolumn{1}{c|}{ Value } \\
\hline$d_{C C R}$ & effective diameter of CCR (not tiled) & $5 \times 10^{-4}[\mathrm{~m}]$ \\
\hline$d_{l}$ & effective diameter of lens (not tiled) & $0.1[\mathrm{~m}]$ \\
\hline$T_{l}$ & effective transmission of lens & 0.8 \\
\hline$T_{f}$ & optical filter transmission & 0.8 \\
\hline$f_{a c t}$ & $\begin{array}{l}\text { fraction of the image sensor pixel area } \\
\text { that is active }\end{array}$ & 0.75 \\
\hline$R$ & detector responsivity & $0.54[\mathrm{~A} / \mathrm{W}]$ \\
\hline$q$ & electron charge & $1.6 \times 10^{-19}[\mathrm{C}]$ \\
\hline$G$ & open-loop voltage gain & 10 \\
\hline$\eta$ & fixed capacitance & $112\left[\mathrm{pF} / \mathrm{cm}^{2}\right]$ \\
\hline$\Gamma$ & FET channel noise factor & 1.5 \\
\hline$g_{m}$ & FET transconductance & $30[\mathrm{mS}]$ \\
\hline$I_{b g}$ & background light current & $5100[\mu \mathrm{A}]$ \\
\hline$k$ & Boltzmann's constant & $1.374 \mathrm{x} 10-23[\mathrm{~J} / \mathrm{K}]$ \\
\hline$T$ & absolute temperature & $298[\mathrm{~K}]$ \\
\hline$F O V$ & field of view & $90^{\circ}$ \\
\hline$A_{r}$ & receiver area & $1.22\left[\mathrm{~cm}^{2}\right]$ \\
\hline$J$ & number of pixels of image sensor & $1141[\mathrm{pixels}]$ \\
\hline
\end{tabular}

Figure 4 illustrates the BER when $R_{b}=1 \mathrm{~kb} / \mathrm{s} ; P_{t}=50 \mu \mathrm{W}, 200 \mu \mathrm{W}$, and $500 \mu \mathrm{W}$ for $h p a=40^{\circ}, 45^{\circ}$, and $50^{\circ}$. We apply the OWC transmitted power lower than or equal to $500 \mu \mathrm{W}$, which is considered safe for the safety standard class 1 operation as explained in Section 3.

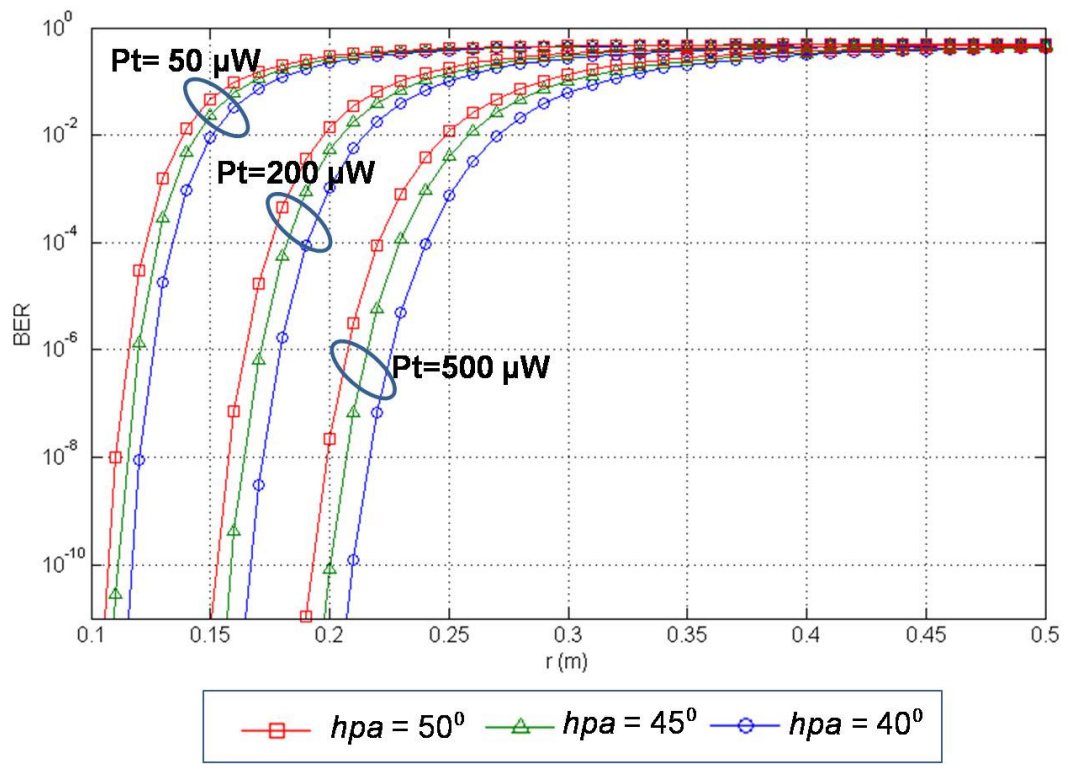

Figure 4. BER when $R_{b}=1 \mathrm{~kb} / \mathrm{s} ; P_{t}=50 \mu \mathrm{W}, 200 \mu \mathrm{W}$, and $500 \mu \mathrm{W}$ 
International Journal of Computer Networks \& Communications (IJCNC) Vol.3, No.5, Sep 2011

As depicted in Figure 4 and in (1) - (3), when hpa is lower, the BER is lower because the intensity at CCR is increased by narrowing the transmitter semi-angle (hpa), which increases $n$. When the intensity at CCR is increased, the average power that propagates back from CCR is also increased and the received photocurrent of $i^{\text {th }}$ pixel at the image sensor is increased. Consequently, SNR is increased as a result of a lower BER. In the same way, when $P_{t}$ is increased, the SNR of OWC is increased and BER is decreased. Figure 4 illustrates that $h p a=40^{\circ}$ provides a lower BER than $h p a=50^{\circ}$, and when $P_{t}=500 \mu \mathrm{W}$, BER is lower than when $P_{t}=50 \mu \mathrm{W}$. In Figure 4, when $R_{b}=1 \mathrm{~kb} / \mathrm{s}$ and the distance between tag and reader is more than $0.25 \mathrm{~m}, B E R_{r e q}$ is not achieved. However, when the data rate is lower, we can attain $B E R_{r e q}$ with a greater distance, as shown in Figure 5.

Figure 5 illustrates BER when $P_{t}=500 \mu \mathrm{W} ; R_{b}=10 \mathrm{~b} / \mathrm{s}, 100 \mathrm{~b} / \mathrm{s}$, and $1 \mathrm{~kb} / \mathrm{s}$. When the data rate is equal to $10 \mathrm{~b} / \mathrm{s}, B E R_{\text {req }}$ is attained when the distance between tag and reader $(r)$ is up to around $0.4 \mathrm{~m}$. However, when the data rate is low, if it is not possible to transmit all the data using OWC, some parts of the information are switched to be transmitted by RFID.

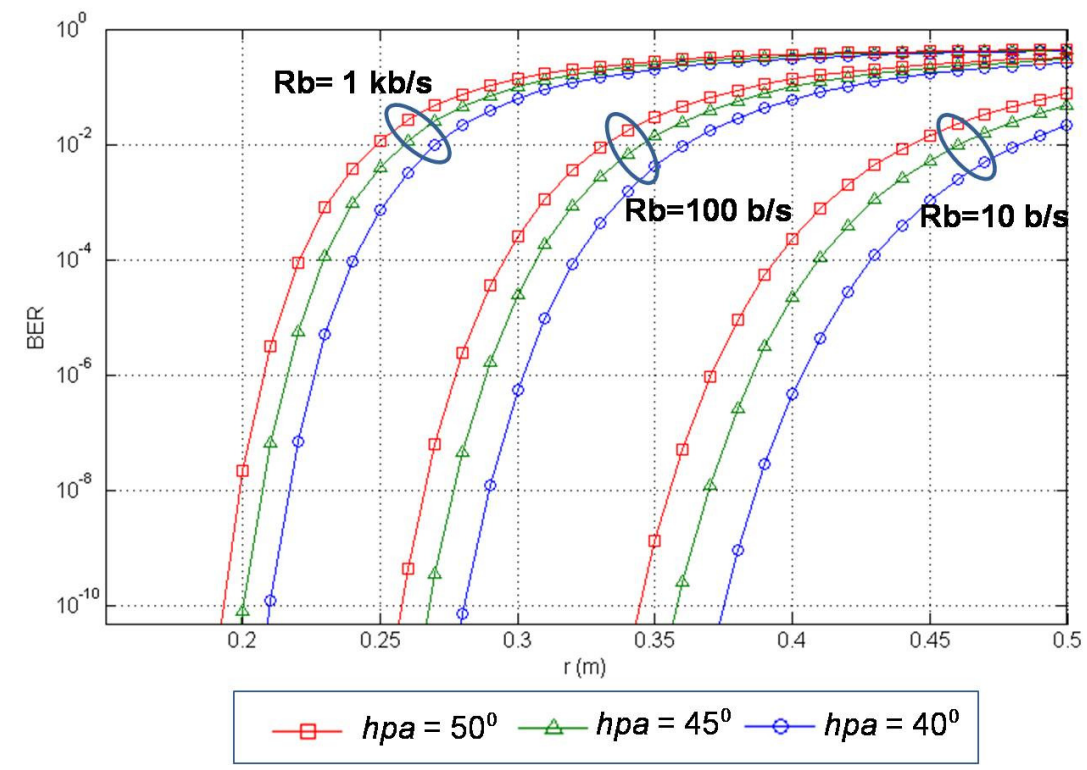

Figure 5. BER when $P_{t}=500 \mu \mathrm{W} ; R_{b}=10 \mathrm{~b} / \mathrm{s}, 100 \mathrm{~b} / \mathrm{s}$, and $1 \mathrm{~kb} / \mathrm{s}$

Figure 6 illustrates BER when $P_{t}=500 \mu \mathrm{W} ; R_{b}=100 \mathrm{bps} ; \theta_{c}=0^{\circ}, 30^{\circ}$, and $60^{\circ}$. As explained in Section $3, \theta_{c}$ is the angle between the laser and the axis of link. We can assume that $\theta_{c}$ is the angle between the tag and reader. When $\theta_{c}=0^{\circ}$, the reader and tag are on-axis, and the interrogating beam comes to the tag at a normal incidence, so a lower BER is achieved. However, in an actual application, the reader and tag may not be on-axis, and the BER would then become higher.

As shown in Figure 6, when $\theta_{c}=30^{\circ}$, the BER is higher than when $\theta_{c}=0^{\circ}$. Moreover, when $\theta_{c}=60^{\circ}$, a low BER is not attained even though the distance between the tag and reader is short (around $0.1 \mathrm{~m}$ ). When $\theta_{c}=60^{\circ}, h p a=50^{\circ}$ has a lower BER than $h p a=40^{\circ}$. Since the higher $h p a$ provides a wider beam, when $\theta_{c}=60^{\circ}$, the optical beam of $h p a=50^{\circ}$ is wider than that of $h p a=40^{\circ}$, and a greater optical signal arrives at the receiver. Therefore, when $h p a=50^{\circ}$, the BER is lower than when $h p a=40^{\circ}$. 


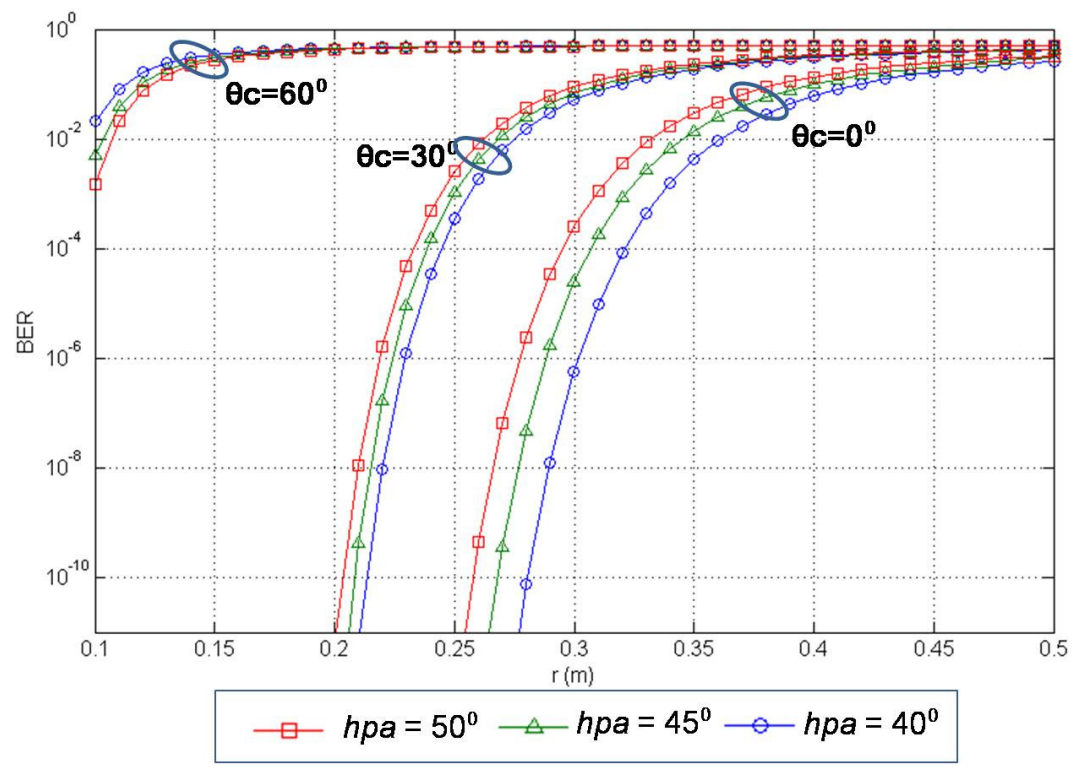

Figure 6. BER when $P_{t}=500 \mu \mathrm{w} ; R_{b}=100 \mathrm{bps} ; \theta_{c}=0^{\circ}, 30^{\circ}$, and $60^{\circ}$

Figure 7 shows the BER of OWC transmission and the BER of the adaptive priority transmission (APT) scheme when $\mathrm{Pt}=500 \mu \mathrm{W}$ and $\theta_{c}=0^{\circ}$. As described in Section 4 , when the BER of the system is more than $B E R_{\text {req }}$ which is equal to $10^{-4}$, the data rate is reduced. Therefore, a BER lower than $10^{-4}$ is achieved nearly all the time. As indicated in Figure 7, although the distance between the tag and reader is around $0.5 \mathrm{~m}$, a lower BER of APT scheme is still achieved because the data rate is reduced.

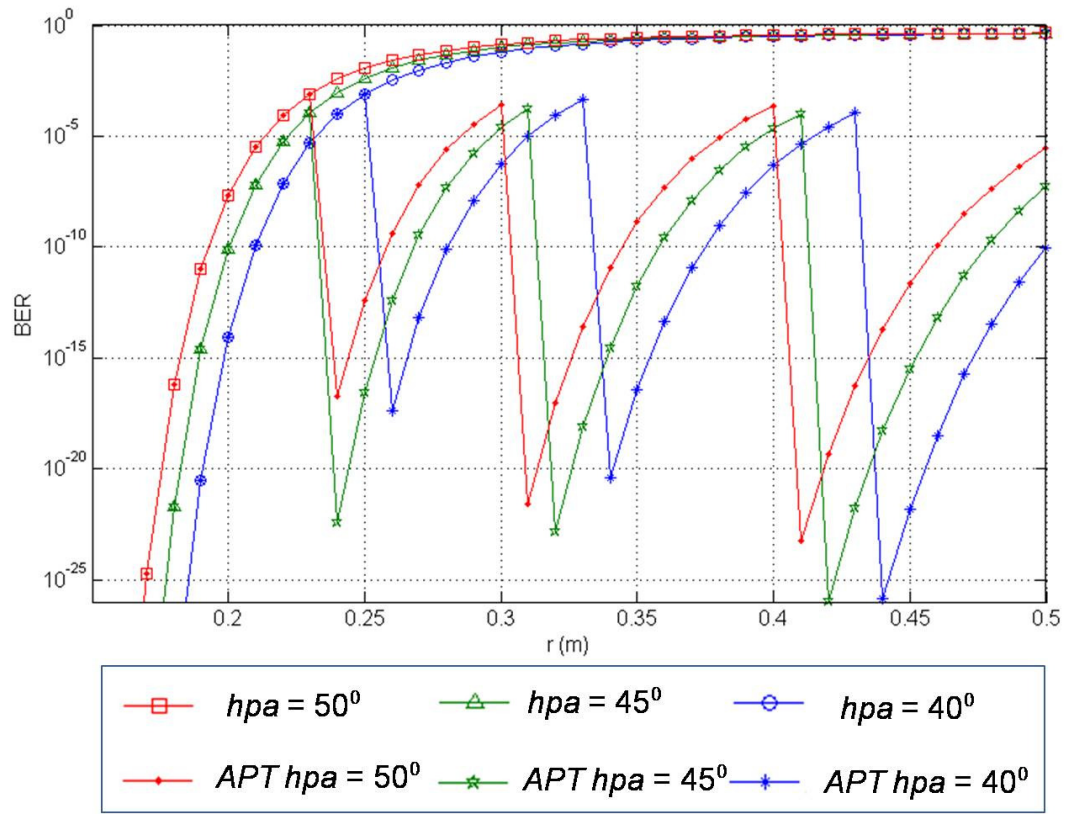

Figure 7. BER when $P_{t}=500 \mu \mathrm{W} ; \theta_{c}=0^{\circ}$ for $\mathrm{OWC}$ transmission and adaptive priority transmission (APT) scheme 
International Journal of Computer Networks \& Communications (IJCNC) Vol.3, No.5, Sep 2011

When $P_{t}=500 \mu \mathrm{W}, \mathrm{r}=0.1 \mathrm{~m}$, and $\theta_{c}=42-60^{\circ}$, the BER of OWC transmission and the BER of the adaptive priority transmission (APT) scheme is illustrated in Figure 8 . When $\theta_{c}$ is increased, the angle between the reader and tag is increased. In order for the OWC transmission beam to arrive at the receiver, a wide beam of the OWC transmitter can achieve a lower BER than a narrow beam. As shown in Figure $8, h p a=50^{\circ}$ provides a lower BER than $h p a=40^{\circ}$. In addition, the APT scheme can ensure that BER is less than $B E R_{\text {req }}$ by adapting the data rate.

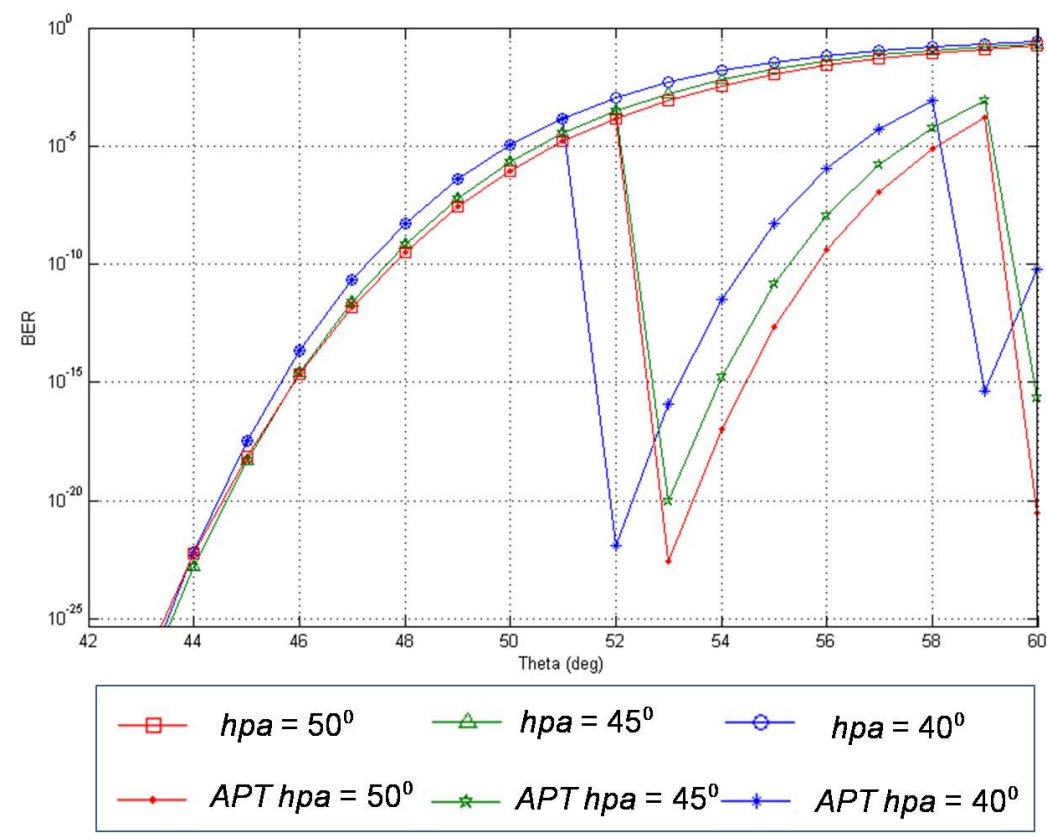

Figure 8. BER when $P_{t}=500 \mu \mathrm{W} ; \mathrm{r}=0.1 \mathrm{~m}$, and $\theta_{c}=42-60^{\circ}$ for $\mathrm{OWC}$ transmission and adaptive priority transmission (APT) scheme

From analytical results, the BER of the OWC transmission system is increased when the distance $(r)$ between the tag and reader is increased, or when the angle $\left(\theta_{c}\right)$ between the tag and reader is increased. We apply an APT scheme so that when $r$ or $\theta_{c}$ is changed, the data rate is adjusted to achieve the required BER.

\section{CONCLUSION}

A hybrid RFID - OWC with an adaptive priority transmission scheme is proposed to improve the security of the RFID system. In our model, security in the physical layer of RFID can be obtained by transmitting confidential information using optical wireless communication. From analytical results, a low BER is attained by increasing the transmitted power, decreasing the distance between the tag and reader, or decreasing the angle between the tag and reader. The adaptive priority transmission scheme is employed to transmit information depending on the level of confidentiality and significance of that information, and also to ensure that the BER is lower than the specific value.

By applying energy harvesting and employing CCR, the RFID tag consumes low energy and can then be a passive tag that does not need any other power sources. Our proposed system, together with other security methods, such as data encryption in the higher layer to strengthen security, can be implemented in high security RFID transmissions, especially in applications 
International Journal of Computer Networks \& Communications (IJCNC) Vol.3, No.5, Sep 2011

that require tag, to transmit sensitive or significant personal information such as date of birth or other personal identification.

\section{REFERENCES:}

[1] Zuo Y, (2010) "Survivable RFID Systems: Issues, Challenges, and Techniques", IEEE trans Systems, Man, and Cybernetics, vol.40, no. 4, pp. 406 - 418.

[2] Y. Yousuf and V. Potdar, (2008) "A Survey of RFID Authentication Protocols", IEEE Conf. on Advanced Information Networking and Applications - Workshops, pp. 1346 - 1350.

[3] D.N. Duc, H. Lee, D.M. Konidala, and K. Kim, (2009) “Open issues in RFID security”, IEEE Conf. on Internet Technology and Secured Transactions, pp. 1 - 5.

[4] Juels A., Molnar D., and Wagner D., (2005) "Security and Privacy Issues in E-passports", IEEE Conf. on SecureComm, pp. $74-88$.

[5] Mahdy A. and Deogun J.S., (2004) "Wireless optical communications: a survey", IEEE Conf. on WCNC, Vol. 4, pp. $2399-2404$.

[6] J.A. Rabadan, S.T. Perez, R. Perez, F.A. Delgado, and M.A. Bacallado, (2005) "Wireless optical spread spectrum communications. Data security improvement in wireless links", IEEE Conf. on ICCST, pp. $168-170$.

[7] W. Noonpakdee, J. Liu, and S. Shimamoto, (2011) "Position based access scheme for indoor optical wireless communication systems," IEEE Conf. on CCNC, pp. 958 - 962.

[8] R.R. Iniguez, S. M. Idrus, and Z. Sun, (2008) Optical Wireless Communications: IR for Wireless Connectivity, Taylor and Francis Group, LLC, Florida.

[9] F. E. Alsaadi and J. M. H. Elmirghani, (2009) “Adaptive Mobile Spot Diffusing Angle Diversity MC-CDMA Optical Wireless System in a Real Indoor Environment”, IEEE Trans on Wireless Communications, Vol. 8, No. 5, pp. 2187 - 2192

[10] Steve Hranilovic (2005), Wireless Optical Communication Systems, Springer Science Business Media, Inc.,Boston.

[11] Bing Jiang, Smith J.R., Philipose M., Roy S., Sundara Rajan K., and Mamishev A.V., (2007) "Energy Scavenging for Inductively Coupled Passive RFID Systems", IEEE trans on Instrumentation and Measurement, vol. 56, no.1, pp. $118-125$.

[12] P. Djahani and J.M. Kahn, (2000) "Analysis of infrared wireless links employing multibeam transmitters and imaging diversity receivers", IEEE Trans on Communications, Vol. 48 , No. 12, pp. 2077 -2088 .

[13] W. Noonpakdee, J. Liu, Kim Dong Hyun, and S. Shimamoto, (2010) "Hybrid RFID employing optical wireless communication," IEEE Conf. on ICWITS, pp. 1- 4.

[14] L. Zhou, J.M. Kahn, and K.S.J. Pister, (2003) "Corner-cube retroreflectors based on structureassisted assembly for free-space optical communication", IEEE Journal of Microelectromechanical Systems, vol.12, No. 3, pp. $233-242$.

[15] Teramoto S. and Ohtsuki T., (2004) "Optical Wireless Sensor Network System Using Corner Cube Retroreflectors," IEEE Conf. on GLOBECOM., vol.2, pp. 1035 - 1039.

[16] T. Komine and M. Nakagawa, (2004) "Fundamental analysis for visible-light communication system using LED lights”, IEEE Trans. on Consum. Electron., vol. 50 , no. 1, pp. 100 - 107.

[17] J. M. Kahn and J. R. Barry, (1977) "Wireless infrared communications," Proc. IEEE, vol. 85, pp. 265-298.

[18] F.E. Alsaadi and J.M.H. Elmirghani, (2010) "High-Speed Spot Diffusing Mobile Optical Wireless System Employing Beam Angle and Power Adaptation and Imaging Receivers", J. Lightw. Technol., vol. 28, no. 16, pp. 2191 - 2206. 
International Journal of Computer Networks \& Communications (IJCNC) Vol.3, No.5, Sep 2011

Authors

Wasinee Noonpakdee received her B.E. degree in electronics engineering from Chulalongkorn University, Thailand, in 1999 and M.ISM. degree in information systems management from Carnegie Mellon University, USA, in 2004. From 1999 to 2003, she worked as an engineer in True Corporation, a telecommunication enterprise in Thailand. From 2004 to 2008, she worked as a computer science officer, Department of Corrections, Ministry of Justice, Thailand. She is currently a Ph.D. student at the Graduate School of Global Information and Telecommunication Studies (GITS), Waseda University, Japan. Her research interests include optical wireless communications, RFID, and wireless sensor networks.

Jiang Liu received her B.E. degree in electronics engineering from Chong Qing University of Technology in 2001 and M.S. degree in information and telecommunications from the Graduate School of Global Information and Telecommunication Studies (GITS), Waseda University, Japan, in 2006. She is currently a Research Associate in Waseda University. Her research interests are optical communication and mobile communication.

Shigeru Shimamoto received the B.E. and M.E. degrees from the University of Electro-Communications, Tokyo, Japan, in 1985 and 1987, respectively. He received the Ph.D. degree from Tohoku University, Sendai, Japan, in 1992. He joined NEC Corporation from 1987 to 1991. From 1991 to 1992, he was a research associate in the University of Electro-Communications, Tokyo, Japan. He was a research associate in Gunma University, Gunma, Japan, from 1992 to 1993. From 1994 to 2000, he was an associate professor in the Graduate School of Global Information and Telecommunication Studies (GITS), Waseda University, Tokyo, Japan. Since 2001, he has been a professor in the Graduate

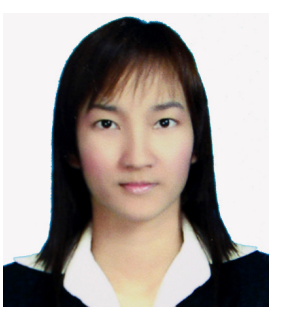
School of GITS, Waseda University. He was a visiting professor of E.E. at Stanford University in 2008. His main fields of research interests include satellite communications, mobile communications, optical wireless communications, ad-hoc networks, sensor networks, and body area networks. 